Supporting Information

\title{
Sacrificial Cyclic Poly(phthalaldehyde) Templates for Low-Temperature Vascularization of Polymer Matrices
}

Mayank Garg, ${ }^{a, b}$ Adam C. Ladd, ${ }^{a, b}$ Jia En Aw, ${ }^{a, c}$ Xiang Zhang, ${ }^{d}$ and Nancy R. Sottos $* a, b$

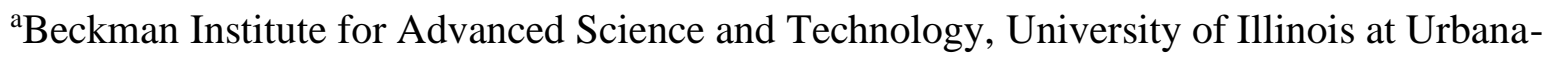

Champaign, Urbana, Illinois, 61801

${ }^{b}$ Department of Materials Science and Engineering, University of Illinois at Urbana-Champaign,

Urbana, Illinois, 61801

${ }^{\mathrm{c}}$ Department of Aerospace Engineering, University of Illinois at Urbana-Champaign, Urbana,

Illinois, 61801

${ }^{\mathrm{d}}$ Mechanical Engineering Department, University of Wyoming, Laramie, Wyoming, 82071

*Email: n-sottos@illinois.edu 


\section{Materials and Instrumentation}

Unless otherwise specified, all reagents were purchased from Sigma-Aldrich and used without further purification. 5-(N-2,3-Dihydroxypropylacetamido)-2,4,6-triiodo-N,N'-bis(2,3dihydroxypropyl)isophthalamide (Histodenz ${ }^{\mathrm{TM}}$ ) was purchased from Sigma Aldrich and used as received. Polytetrafluoroethylene (PTFE) Petri dish liners were purchased from Welch Fluorocarbon Inc. Glass plates and polyurethane gaskets were purchased from McMaster Carr. Glass syringes were purchased from Air-Tite Products Co. Inc. Cyclic poly(phthalaldehyde) (cPPA) was synthesized using a previously reported procedure. ${ }^{1}$ The purified $o$ PA (12 g) was dissolved in anhydrous DCM (96 ml). The solution was transferred to a nitrogen-environment Schlenk Line and cooled to $-78{ }^{\circ} \mathrm{C}$ using an acetone/dry ice bath. Boron trifluoride diethyl etherate ( $2 \mathrm{~mol} \%, 240 \mu \mathrm{l}, 1.8 \mathrm{mmol}$ ) was added dropwise, and the stirred reaction was allowed to equilibrate for two hours at $-78^{\circ} \mathrm{C}$. Pyridine $(400 \mu 1,7.5 \mathrm{mmol})$ was added, and the stirred reaction was allowed to equilibrate for an additional two hours at $-78^{\circ} \mathrm{C}$. The polymer was precipitated dropwise into methanol, vacuum filtered, washed with diethyl ether, and dried for twenty-four hours under vacuum in a freeze drier (Labconco FreeZone 2.5 Liter Benchtop Freeze Dry System).

Molecular weights were obtained via analytical gel permeation chromatography (GPC) on a system composed of a Waters (1515) isocratic high pressure liquid chromatography pump, a Waters (2414) refractive index detector, a Waters (2707) 96-well autosampler, and a series of 4 Waters HR Styragel columns $(7.8 \times 300 \mathrm{~mm}, \mathrm{HR} 1, \mathrm{HR} 3$, HR4, and HR5) in tetrahydrofuran (THF) at $30{ }^{\circ} \mathrm{C}$. The GPC was calibrated using monodisperse polystyrene standards. GPC was performed on the synthesized cPPA solid, films, and fibers. Each sample $(10 \mathrm{mg})$ was fully dissolved in THF $(1 \mathrm{~mL})$, and this solution was injected via a $1 \mathrm{~mL}$ syringe into a $300 \mu \mathrm{L}$ GPC vial through a 0.45 $\mu \mathrm{m}$ polytetrafluoroethylene (PTFE) filter to remove any undissolved particulates. Vials were 
inserted into the GPC autosampler tray and each sample was run with one injection (60 min) according to standard instrument protocol. Number and weight averaged molecular weights $\left(\mathrm{M}_{\mathrm{n}}\right.$ and $\mathrm{M}_{\mathrm{w}}$ ) were calculated using Wyatt's Astra 6 software through integration of the signal peaks obtained from the RI detector using the latest PS calibration. All GPC analyses were performed in triplicates.

Differential scanning calorimetry (DSC) was performed on a TA instruments Discovery DSC 250. For each experiment, a $3 \mathrm{mg}$ sample was measured using an analytical balance (XPE205, MettlerToledo) and transferred into an aluminum hermetic DSC pan at room temperature. The heat flow for each sample was obtained under a constant ramp rate of $10{ }^{\circ} \mathrm{C} / \mathrm{min}$ up to $95^{\circ} \mathrm{C}$.

Visualization of the microchannels within the host matrices was performed by X-ray computed microtomographic $(\mu \mathrm{CT})$ imaging on an Xradia MicroXCT-400 using TXM Controller software. $\mu \mathrm{CT}$ scans were conducted after infiltrating the empty microchannels with Histodenz ${ }^{\mathrm{TM}}$ solution ( $350 \mathrm{mg}$ in $1 \mathrm{~g}$ DI water). $360^{\circ}$ scans were obtained in rotation intervals of $0.4^{\circ}$ with a $4 \mathrm{x}$ objective (5 $\mu \mathrm{m}$ per pixel) at $5 \mathrm{~s}$ exposure times with $40 \mathrm{kV}(200 \mu \mathrm{A}, 8 \mathrm{~W})$ source settings. Scan reconstructions were performed by proprietary software and images were produced via Amira ${ }^{\mathrm{TM}}$ (v. 6.4.0). 


\section{Specimen Fabrication for Vascularization Experiments}

Mass loss calculation for vascularization in PDCPD matrix. The mass loss percentage of cPPA was determined using the following equations:

$$
\text { Mass loss }(\%)=\frac{\Delta M_{C P P A}}{M_{C P P A}} * 100
$$

where $\triangle M_{C P P A}$ represents the mass loss solely due to cPPA evacuation and $M_{C P P A}$ is the estimated mass of the cPPA strip embedded in the pDCPD sample calculated from the mass per unit length of each laser-cut rectangular film,

$$
M_{C P P A}=\frac{M_{i}}{l_{i}} * l_{f}
$$

where $M_{i}$ is the initial mass, $l_{i}$ is the initial length of the laser-cut cPPA strip before it was embedded inside the matrix $\left(l_{i}=20 \mathrm{~mm}\right)$, and $l_{f}$ is the final length of the embedded strip after cutting the pDCPD sample.

The mass loss solely due to cPPA $\left(\Delta M_{C P P A}\right)$ is calculated:

$$
\Delta M_{C P P A}=M_{\text {before }}-M_{\text {after }}-M_{D C P D}
$$

where $M_{\text {before }}$ and $M_{\text {after }}$ are the mass of the entire pDCPD sample containing the cPPA film before and after VaSC, respectively. $M_{D C P D}$ is the mass loss of the similarly sized neat pDCPD matrix at the specific temperature and time being evaluated.

VaSC of cPPA fibers and printed templates in epoxy matrix. Sacrificial templates were embedded in an amine-based epoxy matrix using the same cell-casting procedure as for the pDCPD samples. However, for these samples the glass plates were coated with a PTFE release 
agent (MS-122AD, Miller-Stephenson) before the templates were clamped. The resin EPON 828 (10 g) and the hardener EPIKURE 3233 (4.3 g) were mixed in a paper cup and degassed for 45 min at RT under 12 Torr vacuum (Yamato ADP31 drying oven, Welch 1402 pump). The resinhardener mixture was poured into the glass mold and cured at RT for 24 hours, followed by $35^{\circ} \mathrm{C}$ for 24 hours before cutting with a low-speed wet saw to expose the transverse ends of the sacrificial templates. These samples were then subjected to VaSC in a heated vacuum oven.

VaSC of cPPA fibers in polydimethylsiloxane (PDMS) matrix. Sacrificial templates were embedded in a polydimethylsiloxane (PDMS) matrix using a cell-casting procedure similar to the epoxy matrix. The glass slides were not coated with the PTFE release agent. The PDMS prepolymer and curing agent were mixed in a 10:1 ratio by weight and degassed for 10 minutes before pouring into the mold containing cPPA fibers. The samples were cured at RT for 24 hours, followed by $35^{\circ} \mathrm{C}$ for 24 hours.

VaSC samples were polished using a glycol-based polycrystalline diamond suspension on an Allied MetPrep $3^{\mathrm{TM}}$ with PH-4 ${ }^{\mathrm{TM}}$ Power Head Grinder/Polisher (Allied High Tech Products Inclusive) before optical and electron microscopy. 


\section{Characterization of cPPA Films}

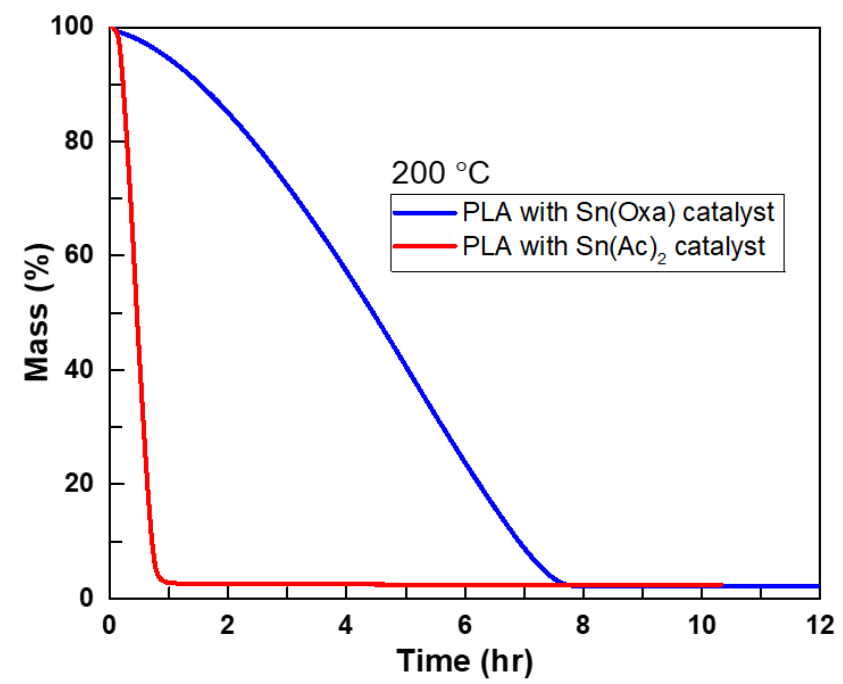

Figure S1. Isothermal TGA on PLA films containing 3 wt. \% tin (II) oxalate ( $\mathrm{Sn}(\mathrm{Oxa})$ ) and 3\% tin (II) acetate $\left(\mathrm{Sn}(\mathrm{Ac})_{2}\right)$ catalyst at $200{ }^{\circ} \mathrm{C} .{ }^{2} \mathrm{PLA}$ depolymerization at $200{ }^{\circ} \mathrm{C}$ can only be achieved using a catalyst and the final mass reflects initial catalyst loading due to the high thermal stability of these catalysts.

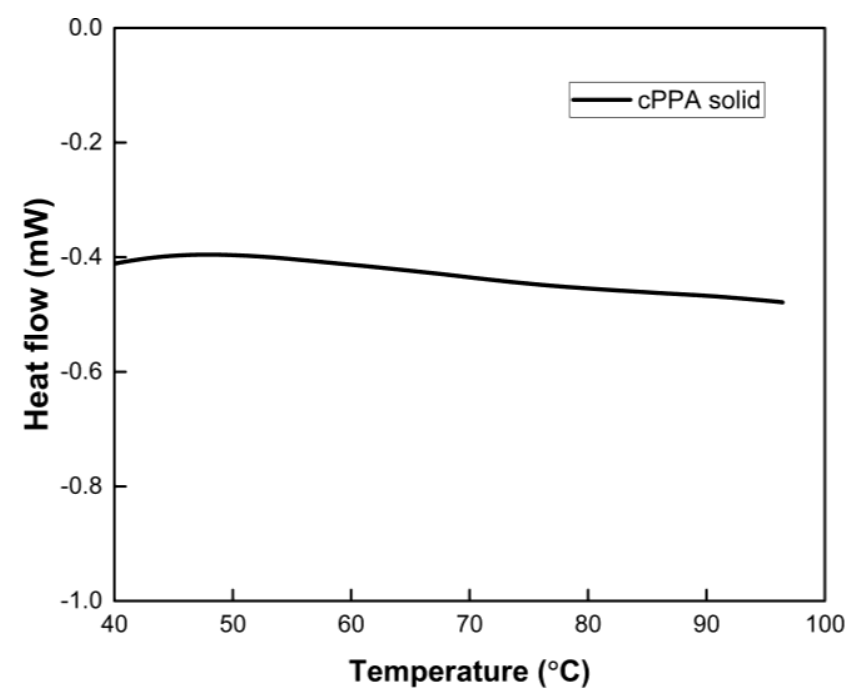

Figure S2. Differential scanning calorimetry of cPPA at $10^{\circ} \mathrm{C} / \mathrm{min}$ showing a lack of observable glass or melt transition temperatures up to $95^{\circ} \mathrm{C}$, which limits cPPA processing to solution-based methods. 
Table S1. Relative molecular weight of cPPA templates after different solvent processing routes using gel permeation chromatography (GPC). Refractive index detector was used to obtain an intensity signal, which was converted to the weight average molecular weight using polystyrene standards. No significant reduction in average molecular weight was observed.

\begin{tabular}{|c|c|c|}
\hline cPPA Sample & $\mathbf{M}_{\mathbf{w}}(\mathbf{k D a})$ & PDI \\
\hline Synthesized solid & $138 \pm 7$ & 1.5 \\
\hline Solution-cast film & $130 \pm 7$ & 1.5 \\
\hline Wet-spun fiber & $128 \pm 7$ & 1.5 \\
\hline
\end{tabular}

Error represents one standard deviation from three different samples.
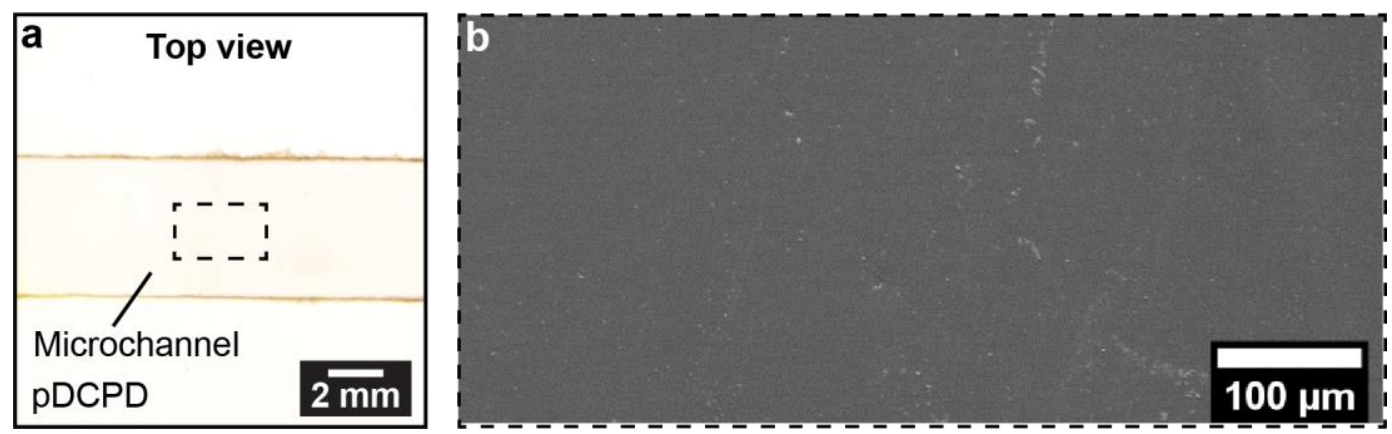

Figure S3. Microchannel characterization after evacuation of a cPPA film from a pDCPD matrix. (a) Optical micrograph and (b) SEM micrograph of a microchannel after VaSC at $100{ }^{\circ} \mathrm{C}$ for three hours.

\section{Characterization of Wet-Spun cPPA Fibers}

DCM and THF were employed as solvents since they are used for synthesizing cPPA. Methanol and water were used as coagulants (i.e., non-solvents) since they are used for precipitating cPPA after synthesis. In an early trial, cPPA dissolved in DCM was coagulated in a methanol bath and the resulting fibers had a bean-type cross-section without any voids (Figure S4a). This finding suggests that the fast diffusion rate of the solvent exceeded the counter-diffusion of methanol to yield a non-uniform fiber cross-section. Subsequent iterations (Figure S4b) confirmed it was difficult to obtain circular fibers with DCM solvent and methanol-water solution as the 
coagulant. The solvent was switched to THF and fibers with a flat cross section were formed during coagulation of cPPA-THF solution in water (Figure S4c), which is likely due to predominant outward diffusion of a miscible solvent in a highly polar coagulant. When water was replaced with methanol to reduce the difference between diffusion and counter-diffusion rates, fibers with a circular cross section and a smooth surface were obtained (Figure S4d). A $50 \%$ reduction in polarity and a $12 \%$ increase in the molar volume of THF compared to DCM likely reduces its diffusion rate and facilitates a gentler coagulation process in methanol to form fibers with a uniform cross section. ${ }^{3,4}$ This hypothesis is also supported by a greater difference between the Hildebrand solubility parameter of methanol and THF (11.2) compared to methanol and DCM $(9.5) .^{5}$

a

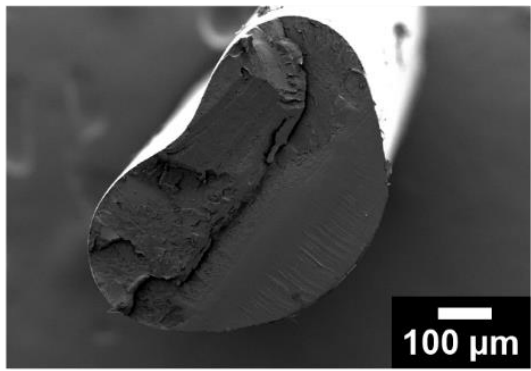

C

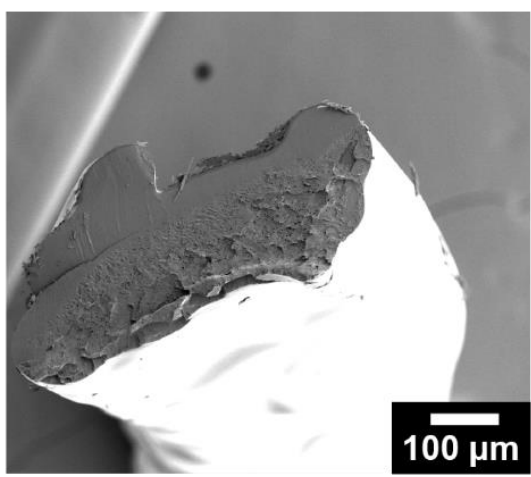

b

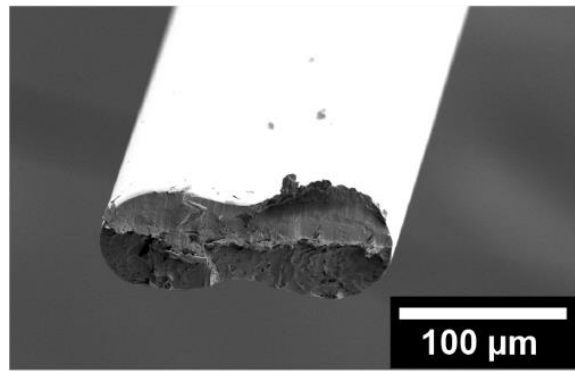

d

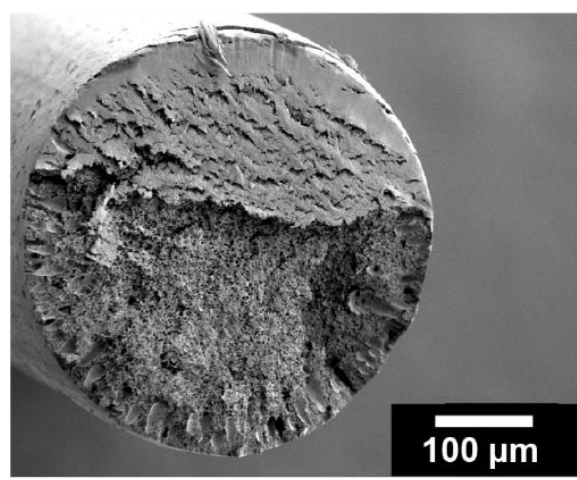

Figure S4. SEM micrographs showing cross section of wet-spun fibers with various solventcoagulant combinations. (a) DCM as solvent and methanol as the coagulant. (b) DCM as solvent and 80:20 (v/v) methanol:water as the coagulant. (c) THF as solvent and water as the coagulant. (d) THF as solvent and methanol as the coagulant. 


\section{Kinetic Depolymerization Model for cPPA Templates in TGA Experiments.}

We leveraged on an optimization scheme to seek parameters that provide the best fit for three isothermal and three dynamic TGA experiments. ${ }^{6}$ The activation energy $(E)$ and pre-exponential factor $(A)$ for this depolymerization model were found to be $89 \mathrm{~kJ} / \mathrm{mol}$ and $4.4 \times 10^{9} \mathrm{~s}^{-1}$, respectively.

$$
\frac{\partial \alpha}{\partial t}=\operatorname{Aexp}\left(-\frac{E}{R T}\right)(1-\alpha)^{n} \alpha^{m}
$$

Table S2. Kinetic parameters obtained using different isothermal and dynamic TGA experiments. Depolymerization model parameters for on PLA films containing $3 \mathrm{wt}$. \% $\mathrm{Sn}(\mathrm{Ac})_{2}$ catalyst are reported from a previous study. ${ }^{2}$

\begin{tabular}{|c|c|c|c|c|}
\hline Sacrificial Polymer & $\boldsymbol{E}\left(\mathrm{kJ} \mathrm{mol}^{-1}\right)$ & $\boldsymbol{A}\left(\mathbf{s}^{-\mathbf{1}}\right)$ & $\boldsymbol{m}$ & $\boldsymbol{n}$ \\
\hline cPPA & 89 & $4.4 \times 10^{9}$ & 0.23 & 0.81 \\
\hline PLA (3\% Sn(Ac) $)$ & 122 & $4.2 \times 10^{10}$ & 0.33 & 0.12 \\
\hline
\end{tabular}

\section{Microchannel Characterization after cPPA Fiber VaSC in pDCPD Matrix}

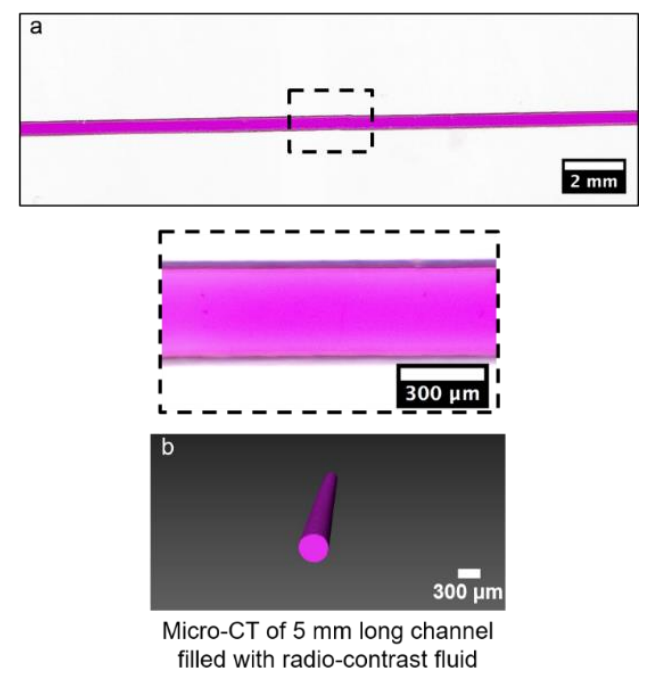

Figure S5. Optical images of a vascularized pDCPD sample: (a) after infiltration with a dye solution and (b) 3D reconstruction of a clear microchannel using X-ray microcomputed tomography (micro-CT) after infiltration with a radiocontrast fluid. 
VII. VaSC with cPPA Templates in Various Matrices with Low $\boldsymbol{T}_{\mathrm{g}}$

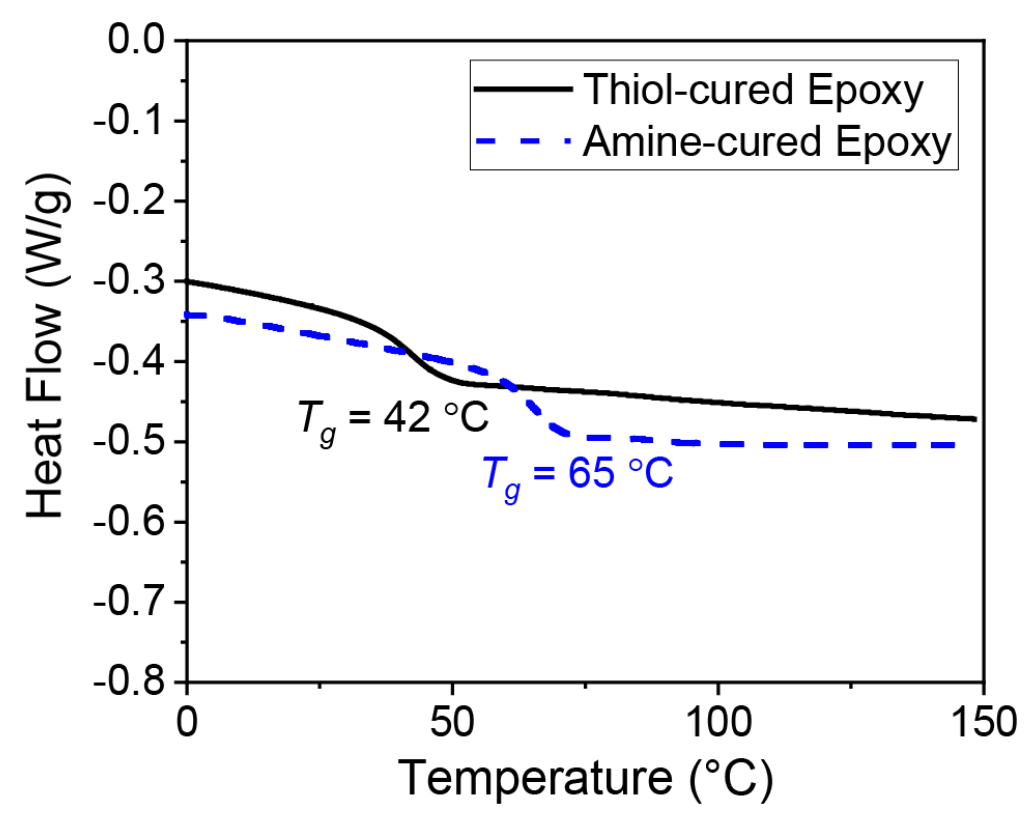

Figure S6. Differential scanning calorimetry on epoxy matrices at $10{ }^{\circ} \mathrm{C} / \mathrm{min}$.
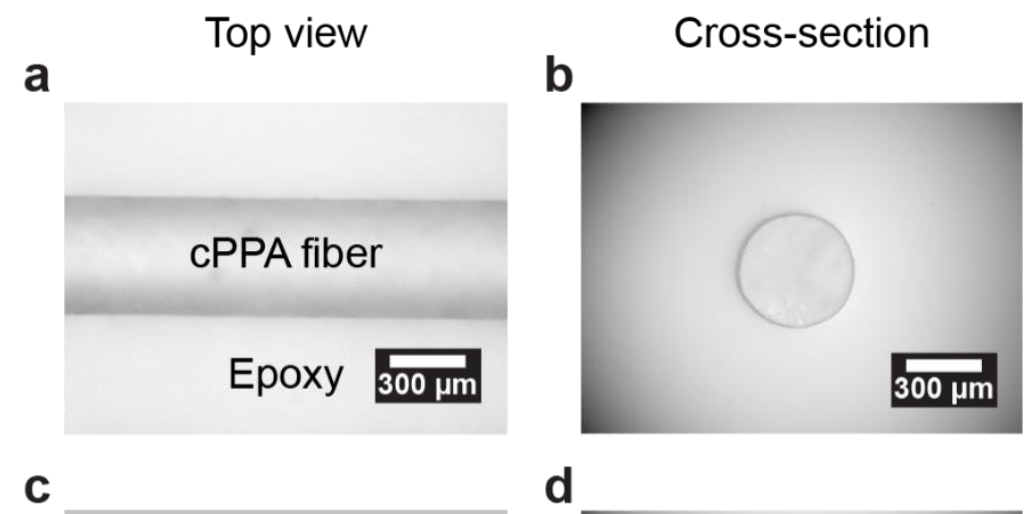

C
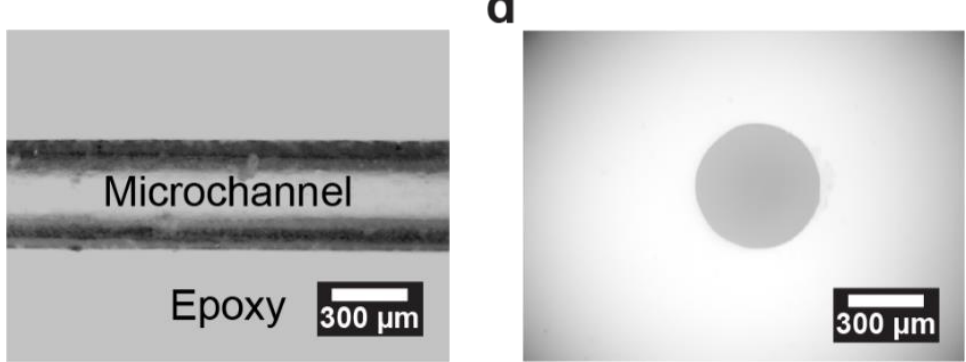

Figure S7. Vascularization of epoxy-based matrix cured using a thiolene-based crosslinker. (a) Top and (b) cross-sectional view of a cPPA fiber embedded in epoxy matrix before VaSC. (c) Top and (d) cross-sectional view of a microchannel in matrix after $\mathrm{VaSC}$ at $110{ }^{\circ} \mathrm{C}$ in $1 \mathrm{~h}$. 

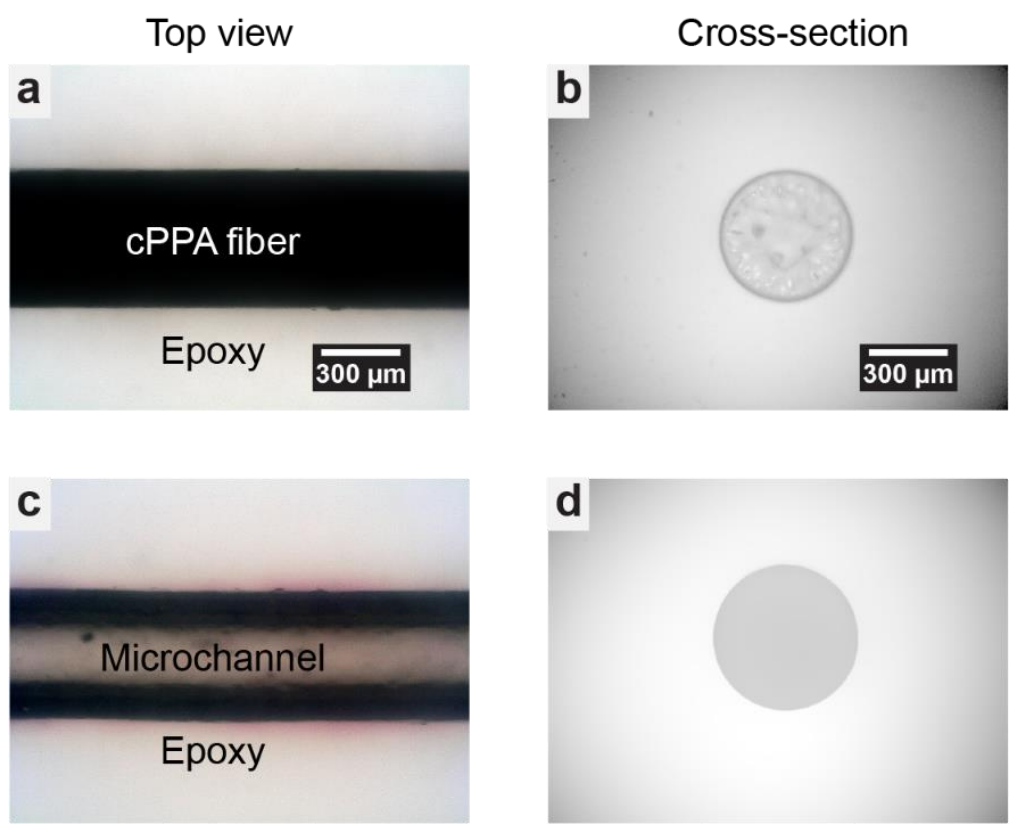

Figure S8. Vascularization of epoxy-based matrix cured using an amine-based crosslinker. (a) top and (b) cross-sectional view of a cPPA fiber embedded in epoxy matrix before VaSC. (c) top and (d) cross-sectional view of a microchannel in matrix after VaSC at $110{ }^{\circ} \mathrm{C}$ in 1 hour.

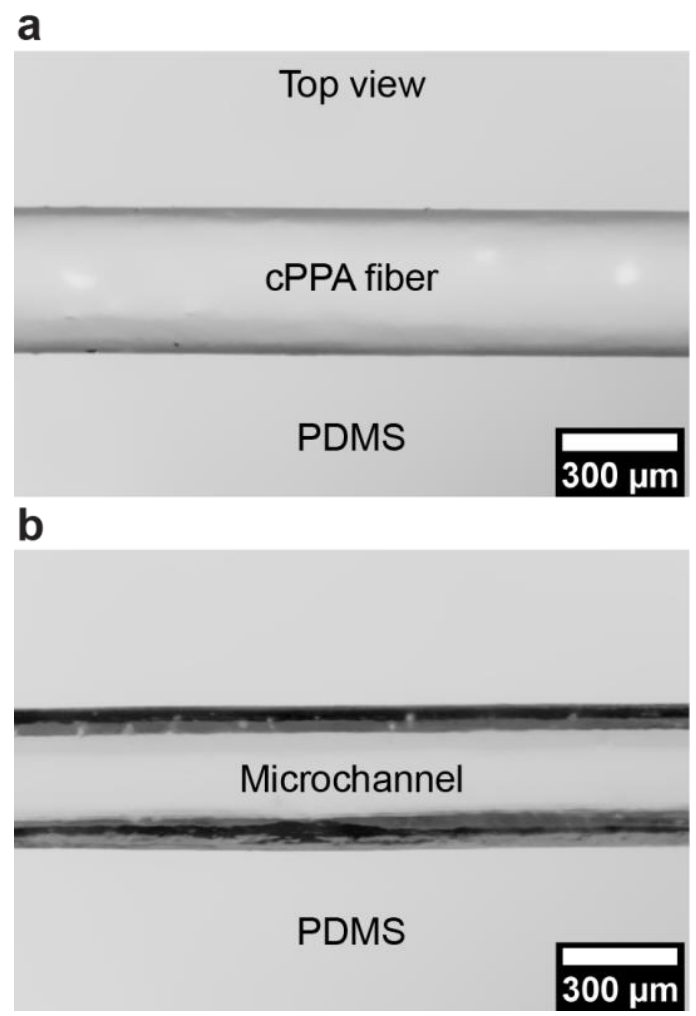

Figure S9. Vascularization of PDMS (Sylgard 184 by Dow Corning) matrix. (a) top view of a cPPA fiber embedded in PDMS matrix before VaSC and (b) top view of a microchannel in matrix after $\mathrm{VaSC}$ at $110^{\circ} \mathrm{C}$ in 1 hour. 

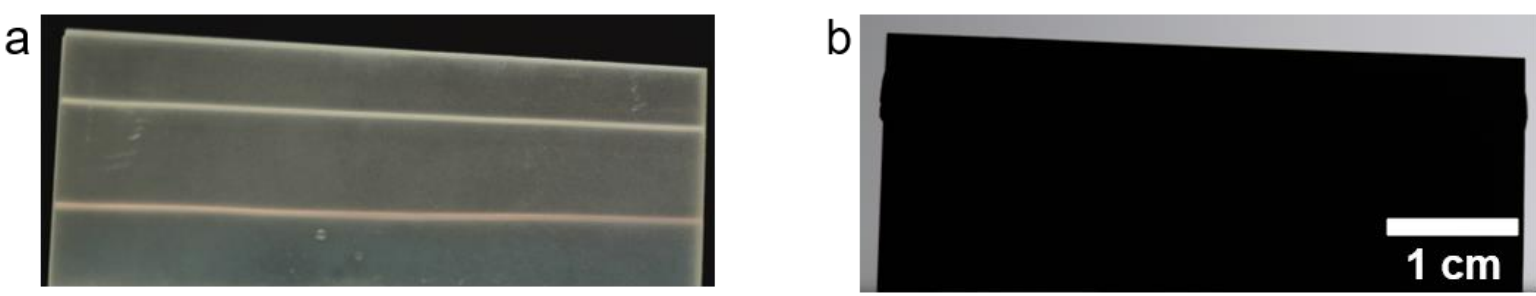

Figure S10. Thermal degradation of epoxy-based matrix cured using a thiol crosslinker after VaSC. (a) top view of a matric with embedded PLA (top) and cPPA (bottom) fibers before VaSC. (b) top view of a charred matrix after $\mathrm{VaSC}$ at $200{ }^{\circ} \mathrm{C}$ for 12 hours.

\section{Energy Estimation for cPPA and PLA VaSC}

Oven power rating: $4.8 \mathrm{~kW}$

Interior volume: $0.8 \mathrm{~m}^{3}$

PLA VaSC cycle: 2 hours from $20{ }^{\circ} \mathrm{C}$ to $200{ }^{\circ} \mathrm{C}$ and then 12 hours at $200{ }^{\circ} \mathrm{C}$

cPPA VaSC cycle: 1 hour from $20^{\circ} \mathrm{C}$ to $110{ }^{\circ} \mathrm{C}$ followed by 1 hour at $110{ }^{\circ} \mathrm{C}$

Table S3. Energy estimation for vascularization cycles of PLA and cPPA

\begin{tabular}{|c|c|c|c|c|c|}
\hline $\begin{array}{c}\text { Sacrificial } \\
\text { Polymer }\end{array}$ & $\begin{array}{c}\text { Heating } \\
\text { Step }\end{array}$ & $\begin{array}{c}\text { Energy Consumption } \\
\text { Rate (kWh/min) }\end{array}$ & $\begin{array}{c}\text { Step time } \\
\text { (min) }\end{array}$ & $\begin{array}{c}\text { Energy } \\
\text { (MJ) }\end{array}$ & $\begin{array}{c}\text { Total } \\
\text { energy (MJ) }\end{array}$ \\
\hline \multirow{2}{*}{ PLA } & Ramp & 0.046 & 120 & 19.9 & \multirow{2}{*}{82.1} \\
\cline { 2 - 5 } & Dwell $^{*}$ & 0.024 & 720 & 62.2 & \\
\hline \multirow{2}{*}{ cPPA } & Ramp & 0.046 & 60 & 9.9 & \multirow{2}{*}{15.1} \\
\cline { 2 - 5 } & Dwell $^{*}$ & 0.024 & 60 & 5.18 & \\
\hline
\end{tabular}

Energy Consumption Ratio $=\frac{82.1}{15.1}=5.4$

* The assumption of similar energy consumption rate during the dwell period for PLA and cPPA cases provides a conservative estimate of energy savings and presents a limitation of our simplified analysis. This rate should be more for the PLA case due to a higher dwell temperature of $200{ }^{\circ} \mathrm{C}$, which would result in higher total energy consumption and a bigger energy consumption ratio. 


\section{References}

(1) Lloyd, E. M.; Hernandez, H. L.; Feinberg, E. C.; Yourdkhani, M.; Zen, E. K.; Mejia, E. B.; Sottos, N. R.; Moore, J. S.; White, S. R. Fully Recyclable Metastable Polymers and Composites. Chem Mater 2018, 31 (2), 398-406. https://doi.org/10.1021/acs.chemmater.8b03585.

(2) Garg, M.; White, S. R.; Sottos, N. R. Rapid Degradation of Poly(Lactic Acid) with Organometallic Catalysts. Acs Appl Mater Inter 2019, 11 (49), 46226-46232. https://doi.org/10.1021/acsami.9b17599.

(3) Knudsen, J. P. The Influence of Coagulation Variables on the Structure and Physical Properties of an Acrylic Fiber. Textile Research Journal 1963, 33 (1), 13-20. https://doi.org/10.1177/004051756303300103.

(4) Ji, B. H. Study on the Mass Transfer Process in Pan Wet-Spinning. Materials Science Forum 2010, 650, 336-342. https://doi.org/10.4028/www.scientific.net/msf.650.336.

(5) Barton, A. F. M. CRC Handbook of Solubility Parameters and Other Cohesion Parameters. 2017. https://doi.org/10.1201/9781315140575.

(6) Das, P.; Tiwari, P. Thermal Degradation Kinetics of Plastics and Model Selection. Thermochimica Acta 2017, 654, 191-202. https://doi.org/10.1016/j.tca.2017.06.001.

(7) Witik, R. A.; Gaille, F.; Teuscher, R.; Ringwald, H.; Michaud, V.; Månson, J.-A. E. Economic and Environmental Assessment of Alternative Production Methods for Composite Aircraft Components. J. Clean. Prod. 2012, 29, 91-102.

https://doi.org/10.1016/j.jclepro.2012.02.028. 